\title{
Methods for inferring neural circuit interactions and neuromodulation from local field potential and electroencephalogram measures
}

\author{
Pablo Martínez-Cañada ${ }^{1,2}$, Shahryar Noei ${ }^{1,3}$ and Stefano Panzeri ${ }^{4,1^{*}}$ (1)
}

\begin{abstract}
Electrical recordings of neural mass activity, such as local field potentials (LFPs) and electroencephalograms (EEGs), have been instrumental in studying brain function. However, these aggregate signals lack cellular resolution and thus are not easy to be interpreted directly in terms of parameters of neural microcircuits. Developing tools for a reliable estimation of key neural parameters from these signals, such as the interaction between excitation and inhibition or the level of neuromodulation, is important for both neuroscientific and clinical applications. Over the years, we have developed tools based on neural network modeling and computational analysis of empirical data to estimate neural parameters from aggregate neural signals. This review article gives an overview of the main computational tools that we have developed and employed to invert LFPs and EEGs in terms of circuit-level neural phenomena, and outlines future challenges and directions for future research.
\end{abstract}

Keywords: Local field potential (LFP), Electroencephalogram (EEG), Neural oscillation, Information theory, Neural network model, Leaky integrate-and-fire (LIF) neuron model, Neuromodulation

\section{Introduction}

Neural activity is often recorded at the level of aggregate electrical signals. These signals are recorded invasively in animals (for example, local field potentials, LFPs, and electrocorticograms, ECoGs $[1,2]$ ) or non-invasively in humans (for example, electroencephalograms, EEG, and magnetoencephalograms, MEG [1, 3-5]). These different aggregate brain signals largely share the same neural sources and have major applications in both scientific research and clinical diagnosis. They are easy to record, capture many circuit-level aggregate phenomena, including key synaptic integrative signals at different

\footnotetext{
*Correspondence: stefano.panzeri@gmail.com; s.panzeri@uke.de ${ }^{4}$ Department of Excellence for Neural Information Processing, Center for Molecular Neurobiology (ZMNH), University Medical Center HamburgEppendorf (UKE), Hamburg, Germany

Full list of author information is available at the end of the article
}

organization levels from mesoscopic to macroscopic brain scales, and can reveal oscillatory activity over a wide range of frequencies [1, 2, 6-9]. However, neural aggregate signals are more difficult to interpret than spiking activity of individual neurons, because they conflate and add together contributions from many complex neural processes $[1,2,6-8]$. It is therefore notoriously difficult to link them to individual neural circuit features. For example, we still cannot interpret simple modulations of EEG/LFP features, such as a change in LFP or EEG oscillatory power, in terms of excitation, inhibition, and their interaction. This hinders us from understanding cognitive computations in humans and animals, understanding the neural underpinnings of brain disorders, and developing effective interventions. Being able to separate contributions of different neural phenomena to LFPs or EEGs, and to quantify how neural parameters change with manipulations of neural circuits or in brain disorders,
Springer Open

(c) The Author(s) 2021. Open Access This article is licensed under a Creative Commons Attribution 4.0 International License, which permits use, sharing, adaptation, distribution and reproduction in any medium or format, as long as you give appropriate credit to the original author(s) and the source, provide a link to the Creative Commons licence, and indicate if changes were made. The images or other third party material in this article are included in the article's Creative Commons licence, unless indicated otherwise in a credit line to the material. If material is not included in the article's Creative Commons licence and your intended use is not permitted by statutory regulation or exceeds the permitted use, you will need to obtain permission directly from the copyright holder. To view a copy of this licence, visit http://creativecommons.org/licenses/by/4.0/. 
will enhance our understanding of how best to use LFPs or EEGs to study brain function and dysfunction.

Over the years, we have developed numerous computational tools to address this challenge. Our approach includes advanced methods to identify meaningful bands in the frequency domain in neural recordings, neural network models to predict key neural phenomena, and computationally guided perturbations of neural activity to causally validate model predictions. This paper summarizes progress achieved by our lab in the interpretation of aggregate electrical signals and introduces new directions and challenges for future research in this field. Since this is an extended review of our work presented as Plenary Talk at the 14th International Conference on Brain Informatics BI 2021 [10], here we have principally focused on describing the computational methods and the results coming from our own Laboratory. We would like, though, to remind the readers of the large number of very important contributions in this field made by many other authors, summarized in recent important reviews $[1,2,5,11,12]$.

\section{Cortical oscillations and their role in neural computation}

Much of our work has been aimed at understanding the neural mechanisms and functions for information processing of brain oscillations captured by LFPs and EEGs. We thus briefly describe some basic features of neural oscillatory activity that are relevant for our review.

Aggregate electrical signals recorded in the cerebral cortex often display prominent oscillatory activity. A large bulk of evidence shows that oscillations seen in neural activity are not simply an epiphenomenon, but are a core mechanism in a variety of cognitive, sensory and information transmission functions [4, 13-24]. Synchronization of neuronal oscillations at different frequencies is a pervasive feature of neuronal activity and is thought to facilitate the transmission and integration of information in the cerebral cortex. Neural aggregate signals have been thus decomposed and interpreted in the frequency domain $[1,6,8]$. Traditionally, neural oscillations have been divided into canonical frequency bands such as the widely used delta $(1-4 \mathrm{~Hz})$, theta $(4-8 \mathrm{~Hz})$, alpha $(8-12 \mathrm{~Hz})$, beta $(15-30 \mathrm{~Hz})$ and gamma $(30-100 \mathrm{~Hz})$ bands. Associations robustly found between band-limited power signals and distinct behavioral states or sensory inputs strongly support the validity of this approach [6, 23, 25-27].

Gamma-band oscillations have received much attention in the last few decades [13-15, 20, 21, 24, 28]. There is a general acceptance that gamma oscillations reflect the interaction between excitation and inhibition in local cortical circuits [20, 21, 29-31]. The power of gamma oscillation encodes information about sensory stimuli, motor and cognitive variables [4, 23, 24, 32-40]. It has been shown that gamma oscillations are also implicated in facilitating or modulating inter-areal or within-area communication [4, 18, 21, 41-46]. Moreover, and of particular importance for the interpretation of neuroimaging experiments in humans, the gamma band is the frequency band that correlates the most with the functional magnetic resonance imaging (fMRI) signal $[47,48]$. The slower theta, alpha and beta rhythms have been involved in many cognitive functions. These slower oscillations have been proposed to mediate top-down perceptual decision processes, encoded in long-range cortical inputs, which could also interact with gammaband synchronization $[4,20,24,49]$. Thus, several cortical rhythms coexist in the cerebral cortex, which are often nested into each other and cooperate to shape brain functions and neuronal information processing [20].

\section{Analytical methods to identify regions of the frequency spectrum capturing different neural phenomena of interest}

Numerous studies have characterized the role of the different frequency bands in brain function. However, the individuation and definitions of the exact boundaries of individual frequency bands are often largely arbitrary, based on heuristic criteria and vary substantially between studies [2]. Thus, a first major problem when trying to infer neural mechanisms from aggregate signals is to provide an objective approach to separate aggregate neural signals into different bands each reflecting a different neural phenomenon, and to establish a correspondence between specific frequency regions of the LFP or EEG power spectrum and the underlying neural mechanisms.

One difficulty in this endeavor is that the average neural power spectrum (over either time epochs or trials) of a typical recording (see Fig. 1A for an example of LFP recordings in visual cortex during naturalistic stimulation) is dominated by a power-law aperiodic component and often lacks easily identifiable oscillatory peaks [23, 50]. This could lead us to think that there is no distinctive structure in the power spectrum and, thus, there is no possibility for a clear and objective separation in frequency bands. However, the average spectrum may mask individual variations that correspond to different processing modalities or functions, especially for complex tasks or during stimulation with naturalistic sensory stimuli.

To capture how individual Fourier frequencies vary their power over time in relation to stimulus variations, we developed an information theoretic algorithm (illustrated in Fig. 2) that quantifies the amount of information about each possible stimulus that is carried by the LFP 
A

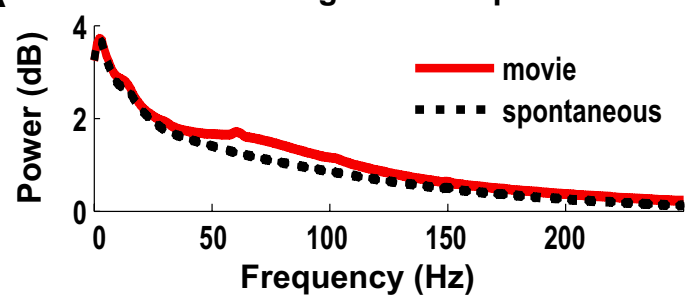

B Information Conveyed by Power Spectrum

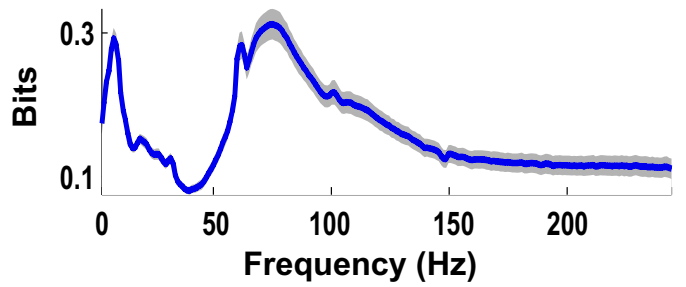

Fig. 1 Comparison of power and information spectra. Data were taken from primary visual cortex of anaesthetized macaques during stimulation with naturalistic movies. A Power spectrum. B Information conveyed by power spectrum. Recomputed from data first published in $[23,48]$

A

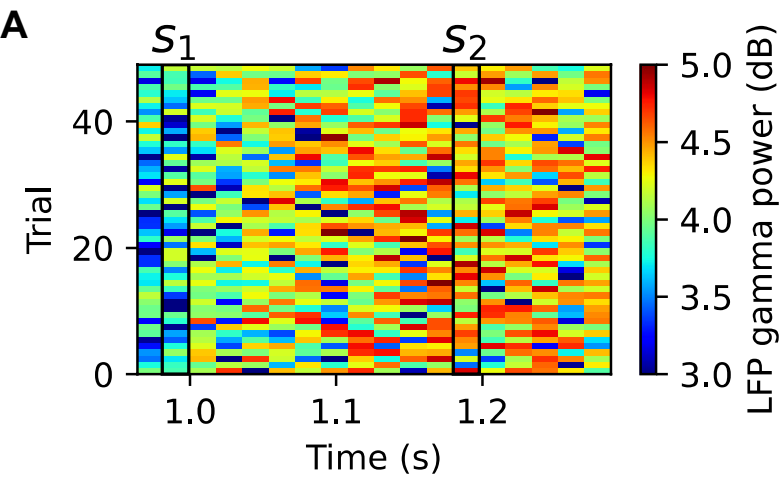

C

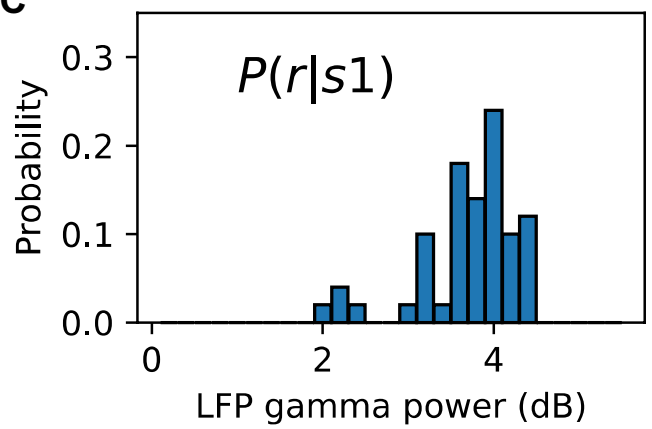

B

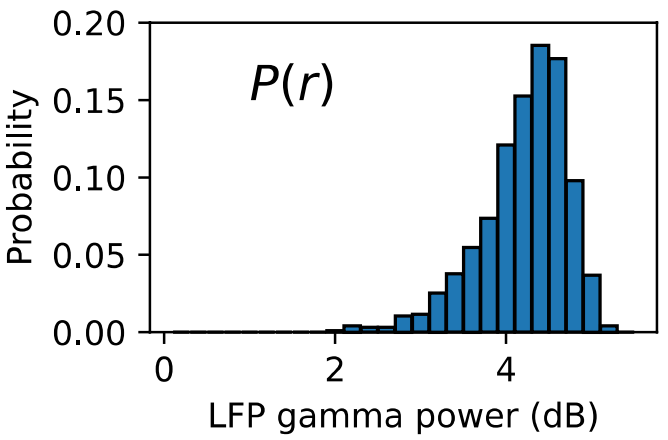

D

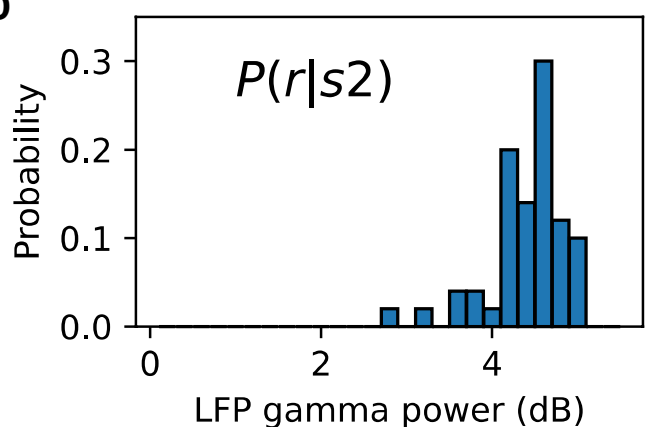

Fig. 2 Illustration of computation of the mutual information carried by LFP power about movie scenes. A Simulation of single-trial LFP power in the gamma band (from 70 to $80 \mathrm{~Hz}$ ) using a sparsely connected recurrent network of excitatory and inhibitory neurons [40]. To simulate periods of low and high LFP power, which approximate the different movie scenes used in the original publication [40], we modulated the external input rate of the model by superposition of a sine wave with frequency $1 \mathrm{~Hz}$ and a constant rate signal. The spectrogram was computed over half a cycle of the sinusoid. Every time window of the spectrogram was considered a different scene $s\left(s_{1}\right.$ and $s_{2}$ are a period of low and high LFP gamma power, respectively). Thus, the probability of each scene $P(s)$ is the inverse of the number of time windows. B Probability distribution $P(r)$ of the LFP gamma power across all trials and scenes. Probability distribution $P(r \mid s)$ of the LFP gamma power across all trials given the presented scenes $s_{1}(\mathbf{C})$ and $s_{2}(\mathbf{D})$

power at a given frequency [40]. The theoretical foundations of information theory (see [51,52]) demonstrate that mutual information is the best measure to capture all possible ways in which a neural signal can carry information about any sensory variable of interest. To create the stimulus set, we divided the presentation time of the movie into different time windows (Fig. 2A), each considered a different stimulus $s$ (in other words, a different movie scene). We computed the information between the stimulus window in the movie $s$ that was being presented and the power of the LFP at a given frequency $f$, as follows:

$$
I\left(S ; R_{f}\right)=\sum_{s} P(s) \sum_{r_{f}} P\left(r_{f} \mid s\right) \log _{2} \frac{P\left(r_{f} \mid s\right)}{P\left(r_{f}\right)},
$$

where $P(s)$ is the probability of presentation of the stimulus window $s$ (here, this is the inverse of the total 
number of time windows in which we divided the movie sequence), $P\left(r_{f} \mid s\right)$ is the probability of observing a power $r_{f}$ at a frequency $f$ in response to the stimulus $s$ in a single trial (Fig. $2 \mathrm{C}$ and $\mathrm{D}$ ), and $P\left(r_{f}\right)$ is the probability of observing the power $r_{f}$ across all trials in response to any stimulus (Fig. 2B).

To facilitate the sampling of response probabilities, the space of power values at each frequency was binned [23]. Information is non-negative and quantifies the average reduction of the uncertainty about the stimulus that can be gained from observing a single-trial neural response. We measured it in units of bits, one bit corresponding to a reduction of uncertainty by a factor of two. Importantly, the quantification of information based on the division of the movie into stimulus windows or scenes without defining which visual feature (e.g., contrast, orientation, etc.) is represented in each movie frame allowed us to capture all information about any possible visual features (including both static image features and their variation from frame to frame) present in the movie.

The information spectrum computed for V1 data during naturalistic stimulation showed a clear structure that was invisible in the average spectrum: there were only two bands that carried stimulus information, a lowfrequency $(1-8 \mathrm{~Hz})$ and a high-frequency gamma band $(60-100 \mathrm{~Hz})$, whereas middle frequencies carried little information (Fig. 1B). It is important to note that the gamma band has been traditionally implicated in the coding of information about specific visual features, such as orientation or contrast of a visual input $[35,37]$. The low-frequency band, to our knowledge, was not implicated in the coding of visual information in V1 by any previous study. This discovery of an extra information channel not considered before was in our view enabled by two key features. First, from an experimental point of view, it was crucial to use a complex dynamics visual stimulus (a movie) that included not only a rich variety of image features from one frame to the next, but also a rich variety of naturalistic temporal dynamics of those features. Second, from a computational point of view, we used a formalism that accounted for all possible sources of information, and, in this way, allowed us to identify the sources of coding of information and neural pathways not considered before. This gives us an example of the potential of using an information theoretic analysis for discovering channels that carry different kinds of neural information and thus need to be included in models as partly different neural pathways.

Extending the information theory approach to the multivariate case of information carried by pairs of frequencies (see [53]) allowed us to characterize specific regions of the information spectrum as belonging to only one or multiple bands. This partition into functionally meaningful bands can be achieved very precisely (and even to the point of individuating the optimal frequency values determining the boundaries between different bands) by quantifying patterns of redundancy or independence between the information carried by different frequencies [48]. For example, if the information carried by one frequency is independent of amplitude variations in another frequency, then these two frequencies probably capture different neural contributions to the LFP. If the two frequencies carry redundant information instead, they likely originate from common neural phenomena. Application of this approach to visual cortical data has revealed three different functional bands in the information spectrum [23]. Frequencies in the gamma $(60-100 \mathrm{~Hz})$ range exhibited high visual information and had large redundancy among them, indicating that neural responses at these frequencies have a common component that is stimulus-driven. The same applies to low frequencies $(1-8 \mathrm{~Hz})$, where there was high redundancy between frequencies. Importantly, low and highfrequency frequencies carried independent information, indicating that they act as independent visual information channels and probably originate from separate neural processes.

Finally, frequencies between 15 and $38 \mathrm{~Hz}$ exhibited high correlations between them but not with stimulus information. Based only on these results of the information theoretic analysis, we hypothesized that signals in this middle frequency range are generated by a common process unrelated to the visual stimuli-for example, a neuromodulatory input [23]. We will discuss in the Section "Perturbation experiments guided by predictions of computational models to study the effect of neuromodulation on cortical oscillations" how this hypothesis could be tested causally by pharmacological intervention.

Importantly, the principles of information theory can be used to understand not only how information is encoded in the oscillatory power or phase of each frequency band, but also how activity in different bands is involved in transmission of information across different neural populations. We used the same recordings of LFP activity in macaque V1 during natural movie stimulation discussed above. Our information theoretic methods (in particular, directed measures of information transfer such as transfer entropy) allowed us to investigate how oscillations of cortical activity in the gamma frequency band may influence dynamically the direction and strength of information flow across different groups of neurons. We found that the local phase of gammaband rhythmic activity exerted a stimulus-modulated and spatially asymmetric directed effect on the firing rate of spatially separated populations within the primary visual cortex [45]. The relationships between gamma 
phases at different sites could be described as a stimulus-modulated gamma-band wave propagating along the spatial directions with the maximal flow of information transmitted between neural populations. We observed that gamma waves changed direction during presentation of different movie scenes, and when this occurred, the strength of information flow in the direction of the gamma wave propagation was transiently reinforced. Given that travelling gamma waves indicated the direction of causation in neural activity, we hypothesized that these shifts were associated to a propagation of gamma oscillations along the horizontal connections of V1. Interestingly, we found support for this hypothesis from the fact that the properties of gamma waves were compatible with known physiological and anatomical properties of lateral connectivity. First, travelling gamma waves had an average propagation speed (approximately $364 \mathrm{~cm} / \mathrm{s}$ ) that was similar in magnitude to the signal propagation speed along axons of excitatory horizontal connections reported in the literature [54-57]. Second, information transfer mediated by gamma waves was quantitatively stronger among pairs with similar orientation preference, compatible with the finding that horizontal connections are more likely among populations with similar orientation preferences [58-60]. These effects were specific to the gamma band and were not found in other low-frequency bands [45]. These results suggest that traveling gamma waves mark and causally mediate the dynamic reconfiguration of functional connections and the transfer of visual information within V1 [45].
Together, these examples show the power of information theoretic approaches to interpret individual frequencies in terms of variations with stimuli or behavioral state and to identify a minimal set of meaningful bands whose origin can then be investigated with the aid of computational models and perturbation experiments, as we illustrate in the next sections.

\section{Mathematical modeling of neural network dynamics}

4.1 Neural network models to identify neural mechanisms for information encoding

The above information theoretic analysis individuated two frequency bands that were shown to carry different channels of visual information. The question that arises is what neural circuit mechanisms are expressed by each band. To address this question, we developed a formalism based on fitting recurrent network models of interacting excitatory and inhibitory point neurons (Fig. 3A) to data. These models reduce the morphology of neurons to a single point in space and their dynamics are described by a set of coupled differential equations that can be solved efficiently numerically and often also analytically. Despite their simplicity, these models have been widely used to describe important properties of cortical microcircuits [61], such as sensory information coding [40, 62], working memory [63, 64], attention [65] or sleep slow waves [66]. In particular, we developed a recurrent network model of leaky integrate-and-fire (LIF) neuronal populations composed of 5000 neurons. Consistent with the

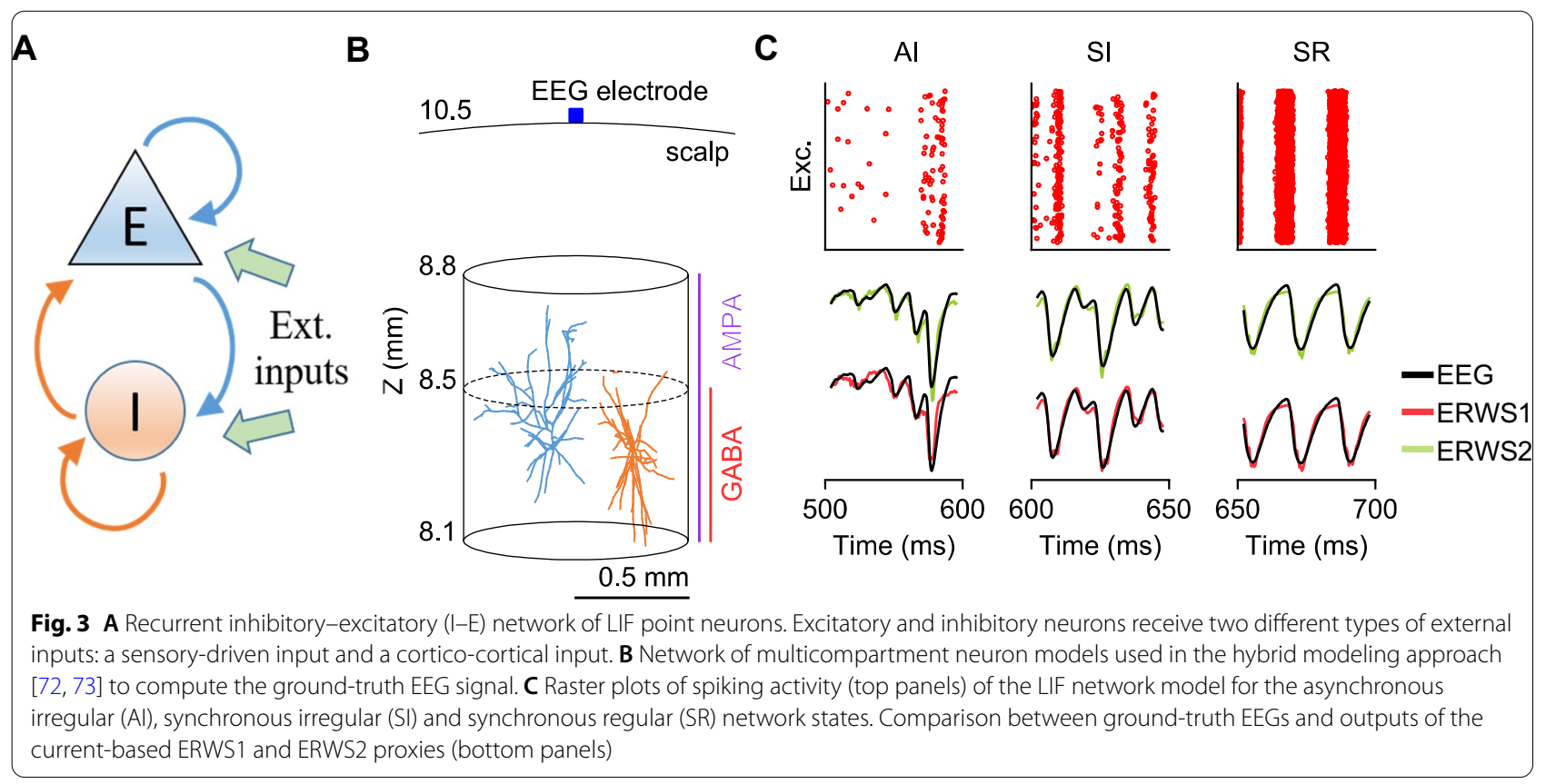


ratio of excitatory and inhibitory neurons found in the cerebral cortex, 4000 neurons were excitatory (i.e., their projections onto other neurons formed AMPA-like excitatory synapses) and 1000 inhibitory (i.e., their projections formed GABA-like synapses), randomly connected with a connection probability between each pair of neurons of 0.2. All neurons in the model receive external inputs (both a sensory-driven thalamic input and a noisy intracortical input) to predict some key aspects of neural activity in primary visual cortex during naturalistic visual stimulation and spontaneous activity $[40,62,67]$.

Specifically, in ref. [40,62], we found that by studying this simulated network we could capture the translation rules between stimulus dynamics and LFP frequency bands. Confirming theoretical results that showed that gamma power in a recurrent network tends to increase with the strength of the input to the network [30], we found that the network encoded the overall strength of the input into the power of gamma-band oscillations generated by inhibitory-excitatory neural interactions. In addition, we found that the network encoded slow dynamic features of the input into slow LFP fluctuations mediated (through entrainment to the inputs) by stimulus-neural interactions. Thus, our recurrent network model could provide evidence for the dual encoding of information in both the low-frequency information channel (carrying temporal information of the dynamics of sensory-driven thalamic inputs) and the gamma-band information channel (reflecting excitatory inhibitory interactions modulated by the strength of thalamic inputs). Interestingly, the model also reproduced other higher order features of the dynamics of visual cortex, including the independence of the information carried by low- and high-frequency information channels when using naturalistic visual stimuli [23], and the crossfrequency coupling between the EEG delta-band phase and gamma-band amplitude [67].

However, our model $[40,62,67]$ could not reproduce the excess in power and the strong within-band correlations observed in real data for the mid-range $(19-38 \mathrm{~Hz})$ band in visual cortex [23]. Our model did not include changes in neural activity induced by neuromodulation, further corroborating the idea that stimulus-independent neuromodulatory factors are needed to model the dynamics of this mid-range band.

\subsection{Realistic computation of field potentials from point-neuron network models}

The above studies compared qualitatively and quantitatively information patterns in neural network models and real data to make inferences about which neural pathway contribute to each frequency band. As demonstrated in ref. [30], this question can be addressed even without having to compute a realistic LFP or EEG from the network models, because basic oscillation properties of the network can be observed both at the level of spiking activity of neurons and at the level of aggregate signals.

We have then begun to investigate the more difficult problem of trying to measure, or to infer, the precise value of microscopic neural parameters, such as the activity of individual classes of neurons within a network, from aggregate activity measures such as EEG or LFP. To obtain a more precise estimation of network parameters, it is necessary to compute a realistic LFP or EEG from these network models of point neurons. However, these neuron models lack a spatial structure, which prevents modelers from being able to compute the spatially separated transmembrane currents that are necessary to generate LFPs and EEGs in real biological networks.

In our initial studies [40, 62, 67], we estimated the LFP and EEG based on the sum of absolute values of synaptic currents from simulation of the network model. Other studies have proposed different approaches to compute extracellular potentials using other variables of the simulation, such as the average membrane potentials $[66,68]$, the average firing rate $[30,69]$ or the sum of all synaptic currents [70, 71].

We then evaluated systematically $[72,73]$ the limitations and caveats of using such ad hoc simplifications to estimate the LFP or EEG from neuron models without spatial structure (i.e., point-neuron models). We compared how well different approximations of field potentials (termed proxies) proposed in the literature reconstructed a ground-truth signal obtained by means of the hybrid modeling approach [72, 74] (Fig. 3B). This approach includes a network of unconnected multicompartment neuron models with realistic three-dimensional (3D) spatial morphologies. Each multicompartment neuron is randomly assigned to a unique neuron in the network of point neurons and receives the same input spikes of the equivalent point neuron. Since the multicompartment neurons are not connected to each other, they are not involved in the network dynamics and their only role is to transform the spiking activity of the point-neuron network into a realistic estimate of the LFP or EEG that is used as the ground-truth signal against which we compared different candidate proxies (Fig. 3C).

We found that a specific weighted sum of synaptic currents from the point-neuron network model, for a specific network state (i.e., asynchronous irregular), performed remarkably well in predicting the LFP [72]. We then extended our study to the EEG [73] by including a head model that approximated the different geometries and electrical conductivities of the head necessary for computing a realistic EEG signal recorded by scalp electrodes. We chose the four-layered spherical head model 
$[75,76]$ that included different layers that represented the brain tissue, cerebrospinal fluid (CSF), skull, and scalp.

We also validated our EEG proxies across the repertoire of network states displayed by recurrent network models [30, 77], namely the asynchronous irregular (AI), synchronous irregular (SI), and synchronous regular (SR) (Fig. 3C). The states generated by the LIF neuron network were produced by systematically varying across simulations the firing rate of the thalamic input $\left(v_{0}\right)$ and the relative strength between inhibitory and excitatory synapses $\left(g=g_{\mathrm{I}} / g_{\mathrm{E}}\right)$. The validation of our proxies for a wide range of values of $g$ and $v_{0}$ is important to solve the inverse modeling approach and to ensure that our proxies can be used to robustly predict these network parameters from the varied shapes of experimentally recorded EEGs (see Sect. 4.3).

We found that a new class of linear EEG proxies, based on a weighted sum of synaptic currents, outperformed previous approaches and worked well under a wide range of network configurations with different cell morphologies, distributions of presynaptic inputs and positions of the EEG electrode. We also evaluated whether our proxies could perform well when combined with a more complex and anatomically detailed human head model: the New York head model [78], which takes into account the folded cortical surface of the human brain. The EEG topographic maps calculated by applying our proxies to the New York head model correctly predicted time traces of the EEG signal at different electrode positions.

\subsection{Changes in excitation-inhibition (E/I) balance in simulated neural aggregate signals}

Our realistic estimations of aggregate signals from simple point-neuron networks allowed us to invert and use these models to estimate some neural parameters of circuit activity that are not directly accessible from the EEG and LFP. For example, we considered how we could use network models to estimate from such recordings the ratio between excitation and inhibition. The theory of neural network models [30] and the empirical electrophysiological data have reported that the E/I ratio has profound effects on the spectral shape of neural activity. Its imbalance has been implicated in neuropsychiatric conditions, including Autism Spectrum Disorder. In ref. [79], we investigated different biomarkers computed on the power spectrum of LFPs and fMRI blood oxygen leveldependent (BOLD) signal that could be used to reliably estimate the E/I ratio. These biomarkers were the exponent of the $1 / \mathrm{f}$ spectral power law, slopes for the low- and high-frequency regions of the spectrum and the Hurst exponent $(\mathrm{H})$. We simulated the LFP (Fig. 4A) and BOLD signal from our recurrent network model, and studied how these biomarkers changed when we manipulated the
E/I ratio by independently varying the strengths of the inhibitory $\left(g_{\mathrm{I}}\right)$ and excitatory $\left(g_{\mathrm{E}}\right)$ synaptic conductances [80]. Part of our results are shown in Fig. 4. A flattening of 1 /f slopes (Fig. 4C) was found in the excitation-dominated region where the $\mathrm{E} / \mathrm{I}$ ratio is shifted in favor of $\mathrm{E}$ than the reference value used previously [40, 62, 67] to capture cortical power spectra. We also observed that $\mathrm{H}$ decreased in the excitation-dominated region (Fig. 4D). However, shifting the E/I balance towards stronger inhibition had a weaker effect on slopes and $\mathrm{H}$. We then validated our model against in vivo chemogenetic manipulations in mice that either increased neurophysiological excitation or silenced the local activity in the network. When modeling effects of chemogenetic manipulations within the recurrent network model, we found that DREADD manipulations that enhanced excitability of pyramidal neurons reduced steepness of the slopes and led to a decrease in $\mathrm{H}$. Then, we used the predictions of our model of how the ratio $g$ between inhibition and excitation affects spectral properties such as slopes and $\mathrm{H}$ (see Fig. 4) to interpret the spectra of resting state fMRI (rsfMRI) in the medial prefrontal cortex (MPFC) of subjects within the autism spectrum disorder. We found that $\mathrm{H}$ was reduced in the MPFC of autistic males but not females, and using our model we interpreted this change in spectral properties as an indicator of increased excitation in males.

\section{Perturbation experiments guided by predictions of computational models to study the effect of neuromodulation on cortical oscillations}

Biophysically realistic computational models and information theoretic methods can be used to generate predictions and test them with suitably designed perturbation experiments. Finding the best strategy to do it is an active topic of research. In what follows, we briefly review our attempts to address this challenge.

As reviewed above, based on our information theoretic analysis, we have proposed that the mid-frequency range (15-38 Hz approx.), which exhibited high correlations within frequency bands but contained little visual information, may reflect a single source of neuromodulatory inputs. We designed a perturbation experiment to test this hypothesis [81]. We recorded the LFP in primary visual cortex (V1) of anesthetized macaques during spontaneous activity and during visual stimulation with naturalistic movies while pharmacologically perturbing dopaminergic neuromodulation by systemic injection of L-DOPA (a metabolic precursor of dopamine). We found that dopaminergic neuromodulation had marked effects on both spontaneous and movie-evoked neural activity. During spontaneous activity, dopaminergic neuromodulation increased the power of the LFP specifically in the 

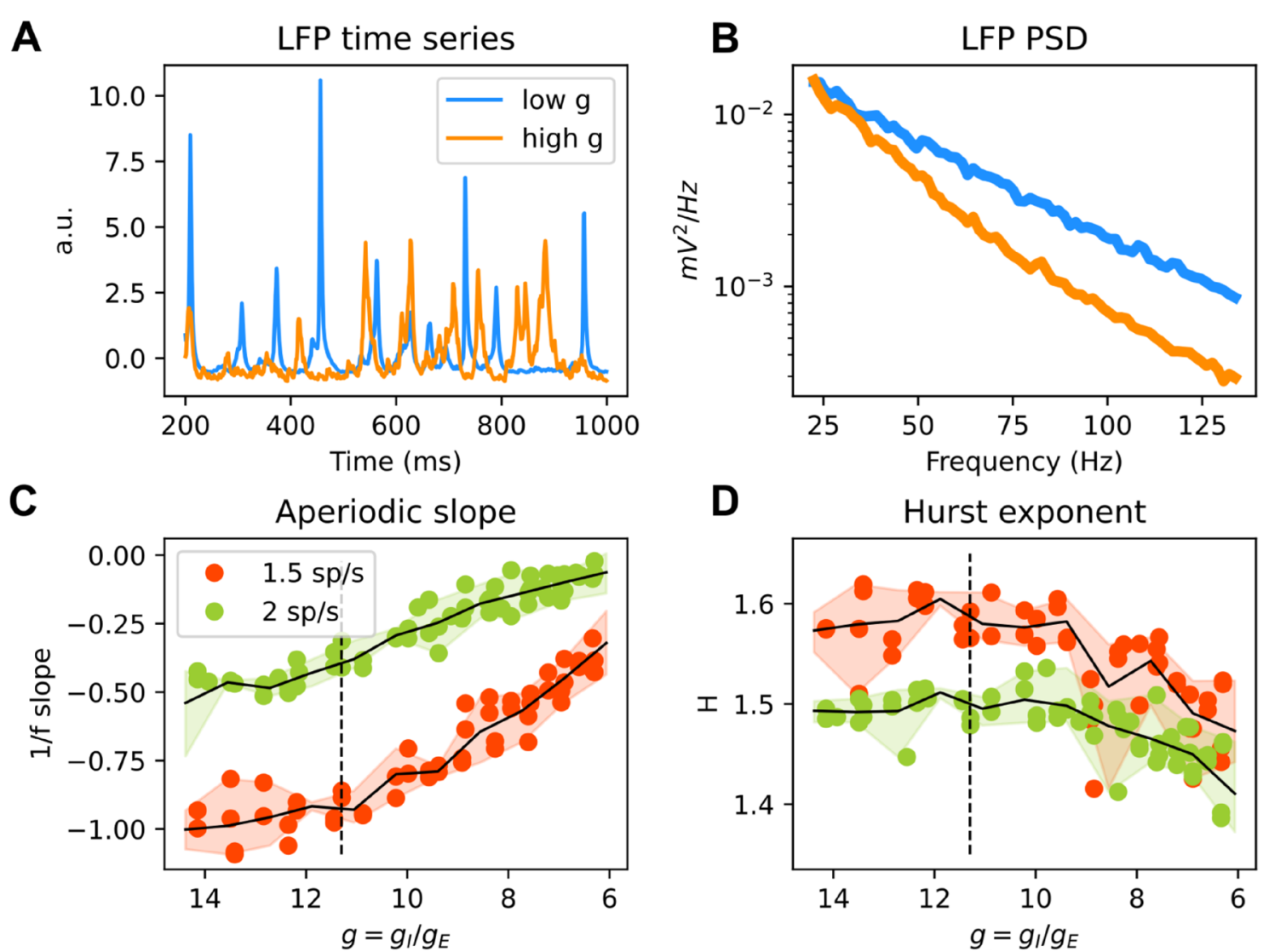

Fig. 4 LFPs $(\mathbf{A})$ and PSDs (B) generated for two different ratios between inhibitory and excitatory conductances $\left(g=g_{/} / g_{E}\right)$. The relationship between $1 / f$ slopes $(\mathbf{C})$ and Hurst exponents $(\mathbf{D})$ are plotted as a function of $g$ for two different firing rates of thalamic input (1.5 and 2 spikes/ second). The reference value of $g$ (which has shown in previous studies to reproduce cortical data well) is represented by a dashed black line. Recomputed and replotted from data published in ref. [79]

19-38 Hz band, suggesting that the power of endogenous visual cortex oscillations in this band can be used as a robust marker of dopaminergic neuromodulation. These results confirmed the hypothesis that we made in earlier work [23] based on information theoretic analysis of field potentials. Moreover, dopamine increased visual information encoding over all frequencies during movie stimulation. The information increase due to dopamine was prominent in the supragranular layers of visual cortex that project to higher cortical area and in the gamma band of LFP power spectrum that has been previously implicated in mediating feedforward information transfer. We concluded that dopamine may promote the readout of relevant sensory information by strengthening the transmission of information from primary to higher areas [81].

These observations, which in our view could not have been made by either computational analyses or blind design of perturbation experiments alone, illustrate the power of effectively combing them.

\section{Conclusion}

Understanding the microcircuit dynamics and computations underlying EEG and LFP features has the potential to allow researchers to make fundamental discoveries about brain function and to effectively use measures extracted from aggregate electrical signals as a reliable biomarker of brain pathologies. In this paper, we have reviewed our approach based on computational modeling and advanced analytical tools of neural network dynamics to interpret neural aggregate signals in terms of neural circuit parameters. We have developed tools to partition the LFP and EEG power spectrum into different meaningful frequency bands and to identify frequency channels and neural pathways that process largely independent and different kinds of neural information. We have shown preliminary work on estimating neural circuit parameters, such as excitation, inhibition and their interaction, from aggregate neural signals. Here we outline some limitations of our approach and the major challenges that we must address in the future. 
In Refs. [72, 73] we have developed accurate LFP and EEG proxies that open up the possibility of computing realistic EEG and LFP predictions from simple network models. These predictions can be then compared to empirical EEGs and LFPs and can be used to estimate neural model parameters. However, to achieve this goal we still need to accomplish several steps. First, we need to develop statistical tools that can infer neural parameters (such as the ratio between excitation and inhibition or properties of network connectivity) from EEG and LFP spectral features by fitting such models to empirically measured spectra. Then, we need to carefully validate the statistical inference approaches on real brain data in which neural circuit parameters can been manipulated by the experimenter, for example by means of chemogenetic manipulations [79]. We could validate the inference algorithm by studying if it is able to predict the type of controlled manipulation that has been applied in each dataset (e.g., whether a manipulation produced an increase or decrease of the E/I balance).

Although we used realistic modeling of neurons and networks, our models do not capture the full complexity of the brain. It would be particularly important to extend our models to include different classes of neurons, such as different types of interneurons. We could include inter-areal interactions between different recurrent networks to generate wider oscillation ranges than the gamma oscillations mostly considered in our work, which would be useful to study the relationship between local oscillations and functional connectivity [82]. It would also be important to model the effects of different kinds of neuromodulators on distributed processing.

In previous work [81], we developed methods to study the effect of global and diffuse patterns of neuromodulation. However, an emergent view [83] is that neuromodulation can be non-global and depend on target specificity and the differentiated spatiotemporal dynamics within brain stem nuclei. It will be important to implement analytical tools to identify first individual ensembles in the locus coeruleus (LC) and to understand then how neural activity of these LC ensembles drive cortical states [84].

Given the above limitations, and although more work is needed to be able to interpret empirical aggregate signals such as EEGs and LFPs in terms of network model parameters and neuromodulation, we expect that future research can build on the encouraging results presented in this paper and lead to a credible, robust and biologically plausible estimation of neural parameters from neural aggregate signals.

\section{Abbreviations}

Al: Asynchronous irregular; BOLD: Blood oxygen level-dependent; ECoG: Electrocorticogram; EEG: Electroencephalogram; fMRI: Functional magnetic resonance imaging; LC: Locus coeruleus; LIF: Leaky integrate-and-fire; LFP: Local field potential; MEG: Magnetoencephalogram; MPFC: Medial prefrontal cortex; rsfMRI: Resting state fMRI; SI: Synchronous irregular; SR: Synchronous regular.

\section{Acknowledgements}

We are most grateful to the organizers and participants of the 14th International Conference on Brain Informatics (BI 2021) for their feedback on the work presented here. We are also deeply grateful to the wonderful colleagues who collaborated with us on these topics over the years, in particular N.K. Logothesis, C. Kayser, O. Eschenko, N. Brunel, G.T. Einevoll, A. Mazzoni, C. Magri, M. Besserve, N.K. Totah, T. Fellin and A. Gozzi.

\section{Authors' contributions}

All authors wrote the paper. All authors read and approved the final manuscript.

\section{Funding}

This project has received funding from the European Union's Horizon 2020 research and innovation programme under the Marie Skłodowska-Curie Grant Agreement No. 893825-ESNECO to P.M.C, the NIH Brain Initiative (Grants U19NS107464 and NS108410 to S.P.), the Simons Foundation (SFARI Explorer 602849 to S.P.), and by the EU FESR-FSE PON “Ricerca \& Innovazione 2014-2020".

\section{Availability of data and materials}

This is a review that contains no new data. Software for simulation of neural network models of spiking point neurons and multicompartment neurons and for computation of EEG proxies can be found at https://github.com/pablo mc88/EEG_proxy_from_network_point_neurons. Software for the information theoretic calculations can be found at https://sicode.eu/results/software.html.

\section{Declarations}

\section{Competing interests}

The authors declare that they have no competing interests.

\section{Author details}

${ }^{1}$ Neural Computation Laboratory, Istituto Italiano Di Tecnologia, Genova and Rovereto, Italy. ${ }^{2}$ Optical Approaches To Brain Function Laboratory, Istituto Italiano Di Tecnologia, Genova, Italy. ${ }^{3} \mathrm{CIMeC}$, University of Trento, Rovereto, Italy. ${ }^{4}$ Department of Excellence for Neural Information Processing, Center for Molecular Neurobiology (ZMNH), University Medical Center HamburgEppendorf (UKE), Hamburg, Germany.

Received: 29 October 2021 Accepted: 29 November 2021

Published online: 15 December 2021

\section{References}

1. Buzsáki G, Anastassiou CA, Koch C (2012) The origin of extracellular fields and currents-EEG, ECoG, LFP and spikes. Nat Rev Neurosci 13(6):407420. https://doi.org/10.1038/nrn3241

2. Einevoll GT, Kayser C, Logothetis NK, Panzeri S (2013) Modelling and analysis of local field potentials for studying the function of cortical circuits. Nat Rev Neurosci 14(11):770-785. https://doi.org/10.1038/nrn35 99

3. Lopes da Silva F (2013) EEG and MEG: relevance to neuroscience. Neuron 80(5):1112-1128. https://doi.org/10.1016/j.neuron.2013.10.017

4. Siegel M, Donner TH, Engel AK (2012) Spectral fingerprints of large-scale neuronal interactions. Nat Rev Neurosci 13(2):121-134. https://doi.org/10. 1038/nrn3137

5. Cohen MX (2017) Where does EEG come from and what does it mean? Trends Neurosci 40(4):208-218. https://doi.org/10.1016/j.tins.2017.02.004

6. Nunez PL, Srinivasan R (2006) Electric fields of the brain: the neurophysics of EEG. Oxford University Press, Oxford. https://doi.org/10.1093/acprof: oso/9780195050387.001.0001

7. Başar E (1980) EEG-brain dynamics: relation between EEG and brain evoked potentials. Elsevier, Amsterdam 
8. Mitra P, Bokil H (2007) Observed brain dynamics. Oxford University Press, Oxford

9. Mahmud M, Vassanelli S (2016) Processing and analysis of multichannel extracellular neuronal signals: state-of-the-art and challenges. Front Neurosci 10:248. https://doi.org/10.3389/fnins.2016.00248

10. Martínez-Cañada P, Noei S, Panzeri S (2021) Inferring neural circuit interactions and neuromodulation from local field potential and electroencephalogram measures. In: Mahmud M, Kaiser MS, Vassanelli S, Dai Q, Zhong N (eds) Brain informatics. Lecture notes in computer science. Springer, Berlin, pp 3-12. https://doi.org/10.1007/978-3-030-86993-9_1

11. Wang X-J, Krystal John H (2014) Computational psychiatry. Neuron 84(3):638-654. https://doi.org/10.1016/j.neuron.2014.10.018

12. Pesaran B, Vinck M, Einevoll GT, Sirota A, Fries P, Siegel M, Truccolo W, Schroeder CE, Srinivasan R (2018) Investigating large-scale brain dynamics using field potential recordings: analysis and interpretation. Nat Neurosci 21(7):903-919. https://doi.org/10.1038/s41593-018-0171-8

13. Buzsaki G, Wang XJ (2012) Mechanisms of gamma oscillations. Annu Rev Neurosci 35:203-225. https://doi.org/10.1146/annur ev-neuro-062111-150444

14. Buzsaki G (2004) Neuronal oscillations in cortical networks. Science 304(5679):1926-1929. https://doi.org/10.1126/science.1099745

15. Jadi MP, Sejnowski TJ (2014) Regulating cortical oscillations in an inhibition-stabilized network. Proc IEEE 102(5):830-842. https://doi.org/ 10.1109/jproc.2014.2313113

16. Wilson HR, Cowan JD (1972) Excitatory and inhibitory interactions in localized populations of model neurons. Biophys J 12(1):1-24. https://doi. org/10.1016/s0006-3495(72)86068-5

17. Whittington MA, Traub RD, Kopell N, Ermentrout B, Buhl EH (2000) Inhibition-based rhythms: experimental and mathematical observations on network dynamics. Int J Psychophysiol 38(3):315-336. https://doi.org/ 10.1016/s0167-8760(00)00173-2

18. Singer W (1999) Neuronal synchrony: a versatile code for the definition of relations? Neuron 24(1):49-65. https://doi.org/10.1016/s0896-6273(00) $80821-1$

19. Wang X-J (2010) Neurophysiological and computational principles of cortical rhythms in cognition. Physiol Rev 90(3):1195-1268. https://doi. org/10.1152/physrev.00035.2008

20. Fries $P(2015)$ Rhythms for cognition: communication through coherence. Neuron 88(1):220-235. https://doi.org/10.1016/j.neuron.2015.09.034

21. Buzsáki G, Schomburg EW (2015) What does gamma coherence tell us about inter-regional neural communication? Nat Neurosci 18(4):484-489. https://doi.org/10.1038/nn.3952

22. Scheeringa R, Fries $P$ (2019) Cortical layers, rhythms and BOLD signals. Neuroimage 197:689-698. https://doi.org/10.1016/j.neuroimage.2017.11. 002

23. Belitski A, Gretton A, Magri C, Murayama Y, Montemurro MA, Logothetis NK, Panzeri S (2008) Low-frequency local field potentials and spikes in primary visual cortex convey independent visual information. J Neurosci 28(22):5696-5709. https://doi.org/10.1523/jneurosci.0009-08.2008

24. Donner TH, Siegel M (2011) A framework for local cortical oscillation patterns. Trends Cogn Sci 15(5):191-199. https://doi.org/10.1016/j.tics.2011. 03.007

25. Lakatos P, Karmos G, Mehta AD, Ulbert I, Schroeder CE (2008) Entrainment of neuronal oscillations as a mechanism of attentional selection. Science 320(5872):110-113. https://doi.org/10.1126/science.1154735

26. Steriade M, Hobson J (1976) Neuronal activity during the sleep-waking cycle. Prog Neurobiol 6(3-4):155-376

27. Ungerleider L, Ray S, Maunsell JHR (2011) Different origins of gamma rhythm and high-gamma activity in macaque visual cortex. PLoS Biol. https://doi.org/10.1371/journal.pbio.1000610

28. Veit J, Hakim R, Jadi MP, Sejnowski TJ, Adesnik H (2017) Cortical gamma band synchronization through somatostatin interneurons. Nat Neurosci 20(7):951-959. https://doi.org/10.1038/nn.4562

29. Bartos M, Vida I, Jonas P (2007) Synaptic mechanisms of synchronized gamma oscillations in inhibitory interneuron networks. Nat Rev Neurosci 8(1):45-56. https://doi.org/10.1038/nrn2044

30. Brunel N, Wang X-J (2003) What determines the frequency of fast network oscillations with irregular neural discharges? I. Synaptic dynamics and excitation-inhibition balance. J Neurophysiol 90(1):415-430. https://doi. org/10.1152/jn.01095.2002
31. Cardin JA, Carlén M, Meletis K, Knoblich U, Zhang F, Deisseroth K, Tsai L-H, Moore Cl (2009) Driving fast-spiking cells induces gamma rhythm and controls sensory responses. Nature 459(7247):663-667. https://doi.org/ 10.1038/nature08002

32. Gray CM, König P, Engel AK, Singer W (1989) Oscillatory responses in cat visual cortex exhibit inter-columnar synchronization which reflects global stimulus properties. Nature 338(6213):334-337. https://doi.org/10.1038/ 338334a0

33. Belitski A, Panzeri S, Magri C, Logothetis NK, Kayser C (2010) Sensory information in local field potentials and spikes from visual and auditory cortices: time scales and frequency bands. J Comput Neurosci 29(3):533-545. https://doi.org/10.1007/s10827-010-0230-y

34. Juergens E, Guettler A, Eckhorn R (1999) Visual stimulation elicits locked and induced gamma oscillations in monkey intracortical- and EEG-potentials, but not in human EEG. Exp Brain Res 129(2):247-259. https://doi. org/10.1007/s002210050895

35. Kayser C, König P (2004) Stimulus locking and feature selectivity prevail in complementary frequency ranges of $\mathrm{V} 1$ local field potentials. Eur J Neurosci 19(2):485-489. https://doi.org/10.1111/j.0953-816X.2003.03122.x

36. Kayser C, Petkov Cl, Logothetis NK (2007) Tuning to sound frequency in auditory field potentials. J Neurophysiol 98(3):1806-1809. https://doi.org/ 10.1152/jn.00358.2007

37. Henrie JA, Shapley R (2005) LFP power spectra in V1 cortex: the graded effect of stimulus contrast. J Neurophysiol 94(1):479-490. https://doi.org/ 10.1152/jn.00919.2004

38. Pesaran B, Pezaris JS, Sahani M, Mitra PP, Andersen RA (2002) Temporal structure in neuronal activity during working memory in macaque parietal cortex. Nat Neurosci 5(8):805-811. https://doi.org/10.1038/nn890

39. Frien A, Eckhorn R, Bauer R, Woelbern T, Gabriel A (2000) Fast oscillations display sharper orientation tuning than slower components of the same recordings in striate cortex of the awake monkey. Eur J Neurosci 12(4):1453-1465. https://doi.org/10.1046/j.1460-9568.2000.00025.x

40. Mazzoni A, Brunel N, Cavallari S, Logothetis NK, Panzeri S (2011) Cortical dynamics during naturalistic sensory stimulations: experiments and models. J Physiol Paris 105(1-3):2-15. https://doi.org/10.1016/j.jphysparis. 2011.07.014

41. Bosman Conrado A, Schoffelen J-M, Brunet N, Oostenveld R, Bastos Andre M, WomelsdorfT, Rubehn B, Stieglitz T, De Weerd P, Fries P (2012) Attentional stimulus selection through selective synchronization between monkey visual areas. Neuron 75(5):875-888. https://doi.org/10.1016/j. neuron.2012.06.037

42. van Kerkoerle T, Self MW, Dagnino B, Gariel-Mathis M-A, Poort J, van der Togt C, Roelfsema PR (2014) Alpha and gamma oscillations characterize feedback and feedforward processing in monkey visual cortex. Proc Natl Acad Sci 111(40):14332-14341. https://doi.org/10.1073/pnas.1402773111

43. Womelsdorf T, Schoffelen J-M, Oostenveld R, Singer W, Desimone R, Engel AK, Fries P (2007) Modulation of neuronal interactions through neuronal synchronization. Science 316(5831):1609-1612. https://doi.org/10.1126/ science. 1139597

44. Fries P (2009) Neuronal gamma-band synchronization as a fundamental process in cortical computation. Annu Rev Neurosci 32(1):209-224. https://doi.org/10.1146/annurev.neuro.051508.135603

45. Kohn A, Besserve M, Lowe SC, Logothetis NK, Schölkopf B, Panzeri S (2015) Shifts of gamma phase across primary visual cortical sites reflect dynamic stimulus-modulated information transfer. PLOS Biol. https://doi. org/10.1371/journal.pbio.1002257

46. Ferro D, van Kempen J, Boyd M, Panzeri S, Thiele A (2021) Directed information exchange between cortical layers in macaque $\mathrm{V} 1$ and $\mathrm{V} 4$ and its modulation by selective attention. Proc Natl Acad Sci 118(12):e2022097118. https://doi.org/10.1073/pnas.2022097118

47. Logothetis NK (2008) What we can do and what we cannot do with fMRI. Nature 453(7197):869-878. https://doi.org/10.1038/nature06976

48. Magri C, Schridde U, Murayama Y, Panzeri S, Logothetis NK (2012) The amplitude and timing of the BOLD signal reflects the relationship between local field potential power at different frequencies. J Neurosci 32(4):1395-1407. https://doi.org/10.1523/jneurosci.3985-11.2012

49. Engel AK, Fries $P$ (2010) Beta-band oscillations - signalling the status quo? Curr Opin Neurobiol 20(2):156-165. https://doi.org/10.1016/j.conb. 2010.02.015

50. Donoghue T, Haller M, Peterson EJ, Varma P, Sebastian P, Gao R, Noto T, Lara AH, Wallis JD, Knight RT, Shestyuk A, Voytek B (2020) Parameterizing 
neural power spectra into periodic and aperiodic components. Nat Neurosci 23(12):1655-1665. https://doi.org/10.1038/s41593-020-00744-x

51. Shannon CE (1948) A mathematical theory of communication. Bell Syst Tech J 27(3):379-423. https://doi.org/10.1002/j.1538-7305.1948.tb01338.x

52. Quian Quiroga R, Panzeri S (2009) Extracting information from neuronal populations: information theory and decoding approaches. Nat Rev Neurosci 10(3):173-185. https://doi.org/10.1038/nrn2578

53. Pola G, Thiele A, Hoffmann KP, Panzeri S (2003) An exact method to quantify the information transmitted by different mechanisms of correlational coding. Network 14(1):35-60. https://doi.org/10.1088/0954-898x/14/1/ 303

54. Bringuier V, Fdr C, Glaeser L, Frégnac Y (1999) Horizontal propagation of visual activity in the synaptic integration field of area 17 neurons. Science 283(5402):695-699. https://doi.org/10.1126/science.283.5402.695

55. Nauhaus I, Busse L, Carandini M, Ringach DL (2008) Stimulus contrast modulates functional connectivity in visual cortex. Nat Neurosci 12(1):7076. https://doi.org/10.1038/nn.2232

56. Grinvald A, Lieke EE, Frostig RD, Hildesheim R (1994) Cortical pointspread function and long-range lateral interactions revealed by real-time optical imaging of macaque monkey primary visual cortex. J Neurosci 14(5):2545-2568. https://doi.org/10.1523/jneurosci.14-05-02545.1994

57. Sato Tatsuo K, Nauhaus I, Carandini M (2012) Traveling waves in visual cortex. Neuron 75(2):218-229. https://doi.org/10.1016/j.neuron.2012.06. 029

58. Stettler DD, Das A, Bennett J, Gilbert CD (2002) Lateral connectivity and contextual interactions in macaque primary visual cortex. Neuron 36(4):739-750. https://doi.org/10.1016/s0896-6273(02)01029-2

59. Roerig B, Chen B (2002) Relationships of local inhibitory and excitatory circuits to orientation preference maps in ferret visual cortex. Cereb Cortex 12(2):187-198. https://doi.org/10.1093/cercor/12.2.187

60. Kisvarday Z (1997) Orientation-specific relationship between populations of excitatory and inhibitory lateral connections in the visual cortex of the cat. Cereb Cortex 7(7):605-618. https://doi.org/10.1093/cercor/7.7.605

61. Einevoll GT, Destexhe A, Diesmann M, Grün S, Jirsa V, de Kamps M, Migliore M, Ness TV, Plesser HE, Schürmann F (2019) The scientific case for brain simulations. Neuron 102(4):735-744. https://doi.org/10.1016/j. neuron.2019.03.027

62. Mazzoni A, Panzeri S, Logothetis NK, Brunel N (2008) Encoding of naturalistic stimuli by local field potential spectra in networks of excitatory and inhibitory neurons. PLoS Comput Biol 4(12):e1000239. https://doi.org/10. 1371/journal.pcbi.1000239

63. Compte A (2000) Synaptic mechanisms and network dynamics underlying spatial working memory in a cortical network model. Cereb Cortex 10(9):910-923. https://doi.org/10.1093/cercor/10.9.910

64. Mongillo G, Barak O, Tsodyks M (2008) Synaptic theory of working memory. Science 319(5869):1543-1546. https://doi.org/10.1126/science. 1150769

65. Deco G, Thiele A (2011) Cholinergic control of cortical network interactions enables feedback-mediated attentional modulation. Eur J Neurosci 34(1):146-157. https://doi.org/10.1111/j.1460-9568.2011.07749.x

66. Hill S, Tononi G (2005) Modeling sleep and wakefulness in the thalamocortical system. J Neurophysiol 93(3):1671-1698. https://doi.org/10.1152/ jn.00915.2004

67. Mazzoni A, Whittingstall K, Brunel N, Logothetis NK, Panzeri S (2010) Understanding the relationships between spike rate and delta/gamma frequency bands of LFPs and EEGs using a local cortical network model. Neuroimage 52(3):956-972. https://doi.org/10.1016/j.neuroimage.2009. 12.040

68. Bazhenov M, Stopfer M, Rabinovich M, Huerta R, Abarbanel HDI, Sejnowski TJ, Laurent G (2001) Model of transient oscillatory synchronization in the locust antennal lobe. Neuron 30(2):553-567. https://doi.org/10.1016/ s0896-6273(01)00284-7

69. Buehlmann A, Deco G (2010) Optimal information transfer in the cortex through synchronization. PLoS Comput Biol. https://doi.org/10.1371/ journal.pcbi.1000934

70. Deco G, Jirsa VK, Robinson PA, Breakspear M, Friston K (2008) The dynamic brain: from spiking neurons to neural masses and cortical fields. PLoS Comput Biol 4(8):e1000092. https://doi.org/10.1371/journal.pcbi.1000092

71. Compte A, Sanchez-Vives MV, McCormick DA, Wang X-J (2003) Cellular and network mechanisms of slow oscillatory activity $(<1 \mathrm{~Hz})$ and wave propagations in a cortical network model. J Neurophysiol 89(5):27072725. https://doi.org/10.1152/jn.00845.2002

72. Mazzoni A, Linden H, Cuntz H, Lansner A, Panzeri S, Einevoll GT (2015) Computing the local field potential (LFP) from integrate-and-fire network models. PLoS Comput Biol 11(12):e1004584. https://doi.org/10.1371/ journal.pcbi.1004584

73. Martinez-Canada P, Ness TV, Einevoll GT, Fellin T, Panzeri S (2021) Computation of the electroencephalogram (EEG) from network models of point neurons. PLoS Comput Biol 17(4):e1008893. https://doi.org/10.1371/journ al.pcbi.1008893

74. Hagen E, Dahmen D, Stavrinou ML, Lindén H, TetzlaffT, van Albada SJ, Grün S, Diesmann M, Einevoll GT (2016) Hybrid scheme for modeling local field potentials from point-neuron networks. Cereb Cortex 26(12):4461-4496. https://doi.org/10.1093/cercor/bhw237

75. Næss S, Halnes G, Hagen E, Hagler DJ, Dale AM, Einevoll GT, Ness TV (2021) Biophysically detailed forward modeling of the neural origin of EEG and MEG signals. Neuroimage. https://doi.org/10.1016/j.neuroimage. 2020.117467

76. Næss S, Chintaluri C, Ness TV, Dale AM, Einevoll GT, Wójcik DK (2017) Corrected four-sphere head model for EEG signals. Front Hum Neurosci. https://doi.org/10.3389/fnhum.2017.00490

77. Brunel N (2000) Phase diagrams of sparsely connected networks of excitatory and inhibitory spiking neurons. Neurocomputing 32-33:307312. https://doi.org/10.1016/s0925-2312(00)00179-x

78. Huang Y, Parra LC, Haufe S (2016) The New York Head-a precise standardized volume conductor model for EEG source localization and tES targeting. Neuroimage 140:150-162. https://doi.org/10.1016/j.neuro image.2015.12.019

79. Trakoshis S, Martínez-Cañada P, Rocchi F, Canella C, You W, Chakrabarti B, Ruigrok ANV, Bullmore ET, Suckling J, Markicevic M, Zerbi V, Consortium MA, Baron-Cohen S, Gozzi A, Lai M-C, Panzeri S, Lombardo MV (2020) Intrinsic excitation-inhibition imbalance affects medial prefrontal cortex differently in autistic men versus women. Elife 9:e55684. https://doi.org/ 10.7554/eLife.55684

80. Martínez-Cañada P, Panzeri S (2021) Spectral properties of local field potentials and electroencephalograms as indices for changes in neural circuit parameters. In: Mahmud M, Kaiser MS, Vassanelli S, Dai Q, Zhong $\mathrm{N}$ (eds) Brain informatics. Lecture notes in computer science. Springer, Berlin, pp 115-123. https://doi.org/10.1007/978-3-030-86993-9_11

81. Zaldivar D, Goense J, Lowe SC, Logothetis NK, Panzeri S (2018) Dopamine is signaled by mid-frequency oscillations and boosts output layers visual information in visual cortex. Curr Biol 28(2):224-235. https://doi.org/10. 1016/j.cub.2017.12.006

82. Canella C, Rocchi F, Noei S, Gutierrez-Barragan D, Coletta L, Galbusera A, Vassanelli S, Pasqualetti M, lurilli G, Panzeri S, Gozzi A (2020) Cortical silencing results in paradoxical fMRI overconnectivity. bioRxiv. https://doi. org/10.1101/2020.08.05.237958

83. Totah NK, Neves RM, Panzeri S, Logothetis NK, Eschenko O (2018) The locus coeruleus is a complex and differentiated neuromodulatory system. Neuron 99(5):1055-1068.e1056. https://doi.org/10.1016/j.neuron.2018.07. 037

84. Noei S, Zouridis IS, Logothetis NK, Panzeri S, Totah NK (2020) Distinct ensembles in the noradrenergic locus coeruleus evoke diverse cortical states. bioRxiv. https://doi.org/10.1101/2020.03.30.015354

\section{Publisher's Note}

Springer Nature remains neutral with regard to jurisdictional claims in published maps and institutional affiliations. 